virulence of Staphylococcus aureus. PLoS One 2009;4(7):e6216.

6. Deng JJ, Xiao GG, Zhu Y, Zhou W, et al. Staphylococcus aureus Nasal Carriage and Its Antibiotic Resistance Profiles in TibetanSchool Children in SouthwestChina. HKJPaediatr (new series) 2014;19:75-8.

7. Lamaro-Cardoso J, de Lencastre H, Kipnis A, Pimenta FC, et al. Molecular epidemiology and risk factors for nasal carriage of Staphylococcus aureus and methicillin-resistant S. aureus in infants attending day care centers in Brazil. J Clin Microbiol 2009;47(12):3991-7.

8. Oliveira DC, de Lencastre H. Multiplex PCR strategy for rapid identification of structural types and variants of the mec element in methicillin-resistant Staphylococcus aureus. Antimicrob Agents Chemother 2002;46(7):2155-61.

9. Lina G, Piémont $Y$, Godail-Gamont F, Bes $M$, et al. Involvement of Panton-Valentine leukocidin-producing
Staphylococcus aureus in primary skin infections and pneumonia. Clin Infect Dis 1999;29(5):1128-32.

10. Stegger M, Andersen PS, Keams A, Pichon B, et al. Rapid detection, differentiation and typing of methicillin-resistant Staphylococcus aureus harbouring either mecA or the new mecA homologue mecA(LGA251). Clin Microbiol Infect 2012;18(4):395-400.

11. Xue H. Pathogenic analysis of Tibetan children in acute low respiratory tract infection in high-altitude areas. Zhongguo Xian Dai Yi Sheng 2015(26):76-8.

12. Xia YL, Ge M, Wang Z. Pathogenic analysis of ventilatorassociated pneumonia in the pediatric intensive care unit in high-altitude areas. Zhongguo Dang Dai Er Ke Za Zhi 2014;16(8):787-90.

13. Airede AI. Neonatal septicaemia in an African city of high altitude. J Trop Pediatr 1992;38(4):189-91.

\title{
Aplicación precoz de presión positiva continua en el tratamiento de la infección respiratoria aguda baja moderada-grave en pacientes menores de 2 años
} Early use of continuous positive airway pressure in the treatment of moderate to severe
acute lower respiratory tract infections among patients younger than 2 years old

\author{
Dra. Laura Figueroa $a^{a}$ Dr. Federico Laffaye ${ }^{a}$
}

\section{RESUMEN}

Objetivo. Analizar las características de los pacientes menores de 2 años que padecieron una infección respiratoria aguda baja moderada-grave tratados precozmente con presión positiva continua en la vía aérea de burbuja y los factores asociados al éxito de la intervención.

Método. Estudio retrospectivo y descriptivo. Niños menores de 2 años internados en Cuidados Intermedios Pediátricos del Hospital Provincial Neuquén entre junio de 2009 y diciembre de 2010. Se aplicó presión positiva continua en la vía aérea de burbuja y se midieron frecuencias cardíacas y respiratorias, puntaje de Tal, saturación y fracción inspirada de oxígeno a las $0,2,6,24$ y $48 \mathrm{~h}$.

Resultados. Se incluyeron 120 pacientes. La mediana de edad fue 3 meses.

La intervención fue exitosa en el $72 \%$ de los casos. A las 2 h, un descenso del $15 \%$ de la frecuencia respiratoria y de 2 puntos en el puntaje de Tal fueron predictores del éxito, con un OR 6,41 (IC 95\%:2,68-15,36) y 9,07 (IC95\%:3,72-22,19), respectivamente.

a. Hospital Provincial Neuquén "Dr. E. Castro Rendón", Unidad de Cuidados Intermedios de Pediatría.

\section{Correspondencia:}

Dra. Laura Figueroa: lauritafigueroa@yahoo.com.ar

Financiamiento: Ninguno.

Conflicto de intereses: Ninguno que declarar.

Recibido: 9-5-2016

Aceptado: 26-12-2016
Conclusiones. La disminución de la frecuencia respiratoria, de la frecuencia cardíaca y del puntaje de Tal a las 2 horas de inicio fueron predictores de éxito.

Palabras clave: CPAP burbuja, bronquiolitis, neumonía, niños.

http:/ / dx.doi.org/10.5546/ aap.2017.277

Texto completo en inglés:

http:/ / dx.doi.org/10.5546/ aap.2017.eng.277

Cómo citar: Figueroa L, Laffaye F. Aplicación precoz de presión positiva continua en el tratamiento de la infección respiratoria aguda baja moderada-grave en pacientes menores de 2 años. Arch Argent Pediatr 2017;115(3):277-281.

\section{INTRODUCCIÓN}

Las infecciones respiratorias agudas bajas (IRAB) representan una importante causa de morbimortalidad en nuestro medio. ${ }^{1}$

Entre las principales entidades clínicas incluidas en las IRAB, se encuentran la bronquiolitis y la neumonía, que son más importantes por su impacto en la mortalidad infantil, ${ }^{2} \mathrm{y}$, sobre estas, se basa el presente trabajo.

\footnotetext{
Colaboradores:

Mario González, Germán Kaltenbach, Viviana Arias y Eugenia Cerda.
} 
La asistencia ventilatoria no invasiva es una alternativa en pacientes con IRAB moderada o grave..$^{3-6}$ Diversas publicaciones mencionan una disminución de los días de hospitalización y una proporción de fracaso menor del 30\%, definida como la necesidad de intubación endotraqueal. ${ }^{7}$

El objetivo de este trabajo fue analizar las características de los pacientes menores de 2 años que padecían una IRAB moderada-grave tratados precozmente con presión positiva continua en la vía aérea (continuous positive airway pressure; $\mathrm{CPAP}$, por sus siglas en inglés) de burbuja y los factores asociados al éxito de la intervención.

\section{POBLACIÓN Y MÉTODOS}

Estudio retrospectivo y descriptivo. Niños menores de 2 años internados entre junio de 2009 y diciembre de 2010. Se aplicó CPAP de burbuja y se midieron frecuencias cardíacas (FC) y respiratorias (FR), puntaje de Tal, saturación y fracción inspirada de oxígeno $\left(\mathrm{FiO}_{2}\right)$ a las $0,2,6$, 24 y 48 h. No se registró gasometría.

Los criterios de inclusión de aplicación de CPAP de burbuja fueron los siguientes:
- Edad de 1 a 24 meses.

- Peso menor de $12 \mathrm{~kg}$.

- Puntaje de Tal mayor de 5 puntos.

- Internación en las unidades de cuidados intermedios de pediatría.

Los criterios de exclusión fueron los siguientes:

- Paro cardiorrespiratorio.

- Inestabilidad hemodinámica a pesar del tratamiento con expansores endovenosos.

- Requerimiento de drogas inotrópicas.

- Ausencia de reflejo nauseoso y / o tusígeno.

La CPAP de burbuja consiste en una interfaz (cánula nasal), una rama inspiratoria y una rama espiratoria conectada a un frasco bajo agua. El paciente respira espontáneamente con un flujo de aire a una presión supraatmosférica, tanto en la inspiración como en la espiración. Se mantiene, así, una presión positiva continua durante todo el ciclo respiratorio.

La CPAP cuenta con un mezclador conectado a una fuente de oxígeno y aire comprimido que se utiliza para suministrar una concentración adecuada de oxígeno inspirado $\left(\mathrm{FiO}_{2}\right)$. El oxígeno humidificado mezclado circula luego

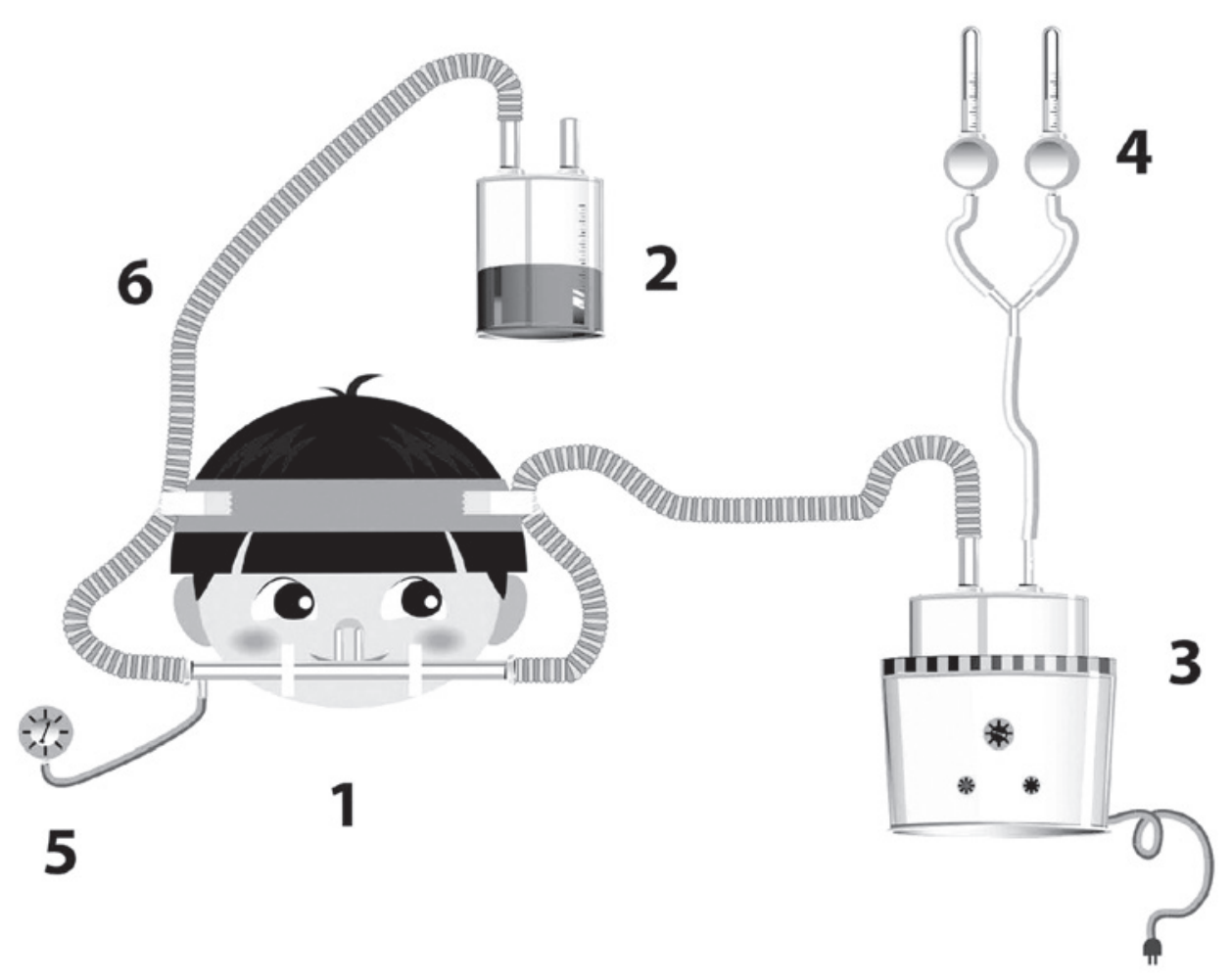

1. Cánula nasal. 2. Frasco bajo agua. 3. Calentador. 4. Fuente aire-oxígeno. 5. Manómetro. 6. Rama espiratoria. 
a través de la tubuladura corrugada. $\mathrm{La} \mathrm{FiO}_{2}$ es calculada sobre la base de los litros de aire y oxígeno suministrados. La mezcla pasa a través de la cánula nasal y la presión en el circuito se mantiene por inmersión del extremo distal del tubo espiratorio en agua. La profundidad a la que el tubo se sumerge bajo el agua determina la presión generada en las vías respiratorias del paciente (Figura 1).

Previamente a la colocación de la interfaz nasal, se aspiraron las secreciones de la vía aérea $\mathrm{y}$, si se lo creía conveniente, se administraba una dosis de sedante (hidrato de cloral o benzodiacepinas).

Se utilizaron cánulas HUDSON RCI-CPAP System, USA, con tamaños del 1 al 5. La CPAP de burbuja se conectó, en el inicio, con un valor de $5 \mathrm{~cm}$ de agua y, eventualmente, se aumentaba en forma progresiva hasta $8 \mathrm{~cm}$ de agua, aunque, según criterio del médico tratante, podía iniciarse con mayor presión. Asimismo, la $\mathrm{FiO}_{2}$ inicial fue de $100 \%$ y luego se descendió según la saturación del paciente.

Se registraron comorbilidades: cardiopatías congénitas, prematurez, desnutrición, displasia broncopulmonar y alto riesgo social (necesidades básicas insatisfechas).

Se buscaron complicaciones, como distención abdominal, lesiones por apoyo y neumotórax.

Además, se registraron peso, edad, sexo, diagnóstico, estudios virológicos (inmunofluorescencia indirecta-IFI-, reacción en cadena de la polimerasa - polymerase chain reaction; PCR, por sus siglas en inglés-), cultivos, administración de sedantes, alimentación, tiempo de estadía con CPAP de burbuja y tiempo de estadía en la Unidad de Cuidados Intensivos Pediátricos (UCIP).

El éxito de la CPAP se evaluó mediante el descenso del 15\% de la FR con respecto a la previa $\mathrm{y}$ un descenso de 2 puntos en el puntaje de Tal.

El fracaso de la CPAP se definió por falta de descenso de puntaje de Tal (FC, FR, tiraje, sibilancias) y / o desaturación (por debajo de 90\%) y/o alteración del sensorio a criterio del médico tratante.

Los criterios para retirar la CPAP de burbuja fueron CPAP de $4 \mathrm{~cm}$ de $\mathrm{H}_{2} \mathrm{O}, \mathrm{FiO}_{2}$ menor de $40 \%$, puntaje de Tal 4.

Para la descripción de la muestra, se utilizaron descriptivos simples, como media, mediana, desviación estándar (DE) y rango. Se calcularon los porcentajes correspondientes a las categorías de las variables cualitativas y se compararon con pruebas chi cuadrado $\left(\chi^{2}\right)$ de independencia para determinar la asociación entre las variables, considerando un nivel de significancia menor del $5 \%$. Se analizaron los cocientes de chance en los casos posibles (odds ratio). Asimismo, se aplicaron gráficos box-plot para describir el comportamiento de las variables cuantitativas.

Para determinar puntos de corte en variables

TABla 1. Características basales de la muestra y de cada grupo según el éxito o la falla. La edad está expresada en mediana y rango; el resto de las variables, en media \pm desviación estándar

\begin{tabular}{|c|c|c|c|c|}
\hline & Todos $(n=120)$ & Grupo de éxito $(n=86)$ & Grupo de fracaso $(n=34)$ & p \\
\hline Edad (meses); X (rango) & $3(1-22)$ & $6,08(2,3-12,45)$ & $5,4(2,9-12)$ & $>0,05$ \\
\hline Femenino & 65 & 41 & 24 & \\
\hline Peso (kg) & $6,49 \pm 2,24$ & $6,55 \pm 2,17$ & $6,35 \pm 2,43$ & \\
\hline FR basal & $68,75 \pm 12,71$ & $67,57 \pm 12,61$ & $71,74 \pm 12,65$ & \\
\hline FC basal & $167,14 \pm 23,05$ & $164,42 \pm 23,71$ & $174,03 \pm 19,99$ & \\
\hline $\mathrm{FiO}_{2}$ basal $(\%)$ & $89,98 \pm 3,13$ & $89,74 \pm 3,36$ & $90,59 \pm 2,39$ & \\
\hline Saturación de pulso & $97,39 \pm 4,09$ & $97,42 \pm 4,53$ & $97,32 \pm 2,69$ & \\
\hline Puntaje de Tal & $9,26 \pm 1,79$ & $9,17 \pm 1,98$ & $9,47 \pm 1,19$ & \\
\hline Bronquiolitis & 55 & 42 & 13 & \\
\hline Neumonía & 65 & 44 & 21 & \\
\hline \multicolumn{5}{|c|}{ Cuadro clínico predisponente } \\
\hline Prematurez & $29(24 \%)$ & $23(79 \%)$ & $6(21 \%)$ & $>0,05$ \\
\hline Desnutrición & $11(9,16 \%)$ & $8(73 \%)$ & $3(27 \%)$ & \\
\hline Displasia broncopulmonar & $6(5 \%)$ & $5(83 \%)$ & $1(17 \%)$ & \\
\hline Cardiopatía congénita & $8(7 \%)$ & $5(62,5 \%)$ & $3(37,5 \%)$ & \\
\hline Alto riesgo social & $14(12 \%)$ & $8(57 \%)$ & $6(43 \%)$ & \\
\hline
\end{tabular}

FR: frecuencia respiratoria; FC: frecuencia cardíaca; $\mathrm{FiO}_{2}$ : fracción inspirada de oxígeno. 
predictivas de falla de la CPAP de burbuja, se utilizaron curvas de sensibilidad y especificidad. Se emplearon curvas de característica operativa del receptor (receiver operating characteristic curve; ROC, por sus siglas en inglés) y su test correspondiente para determinar variables con mejor discriminación de falla de la CPAP de burbuja.

El protocolo de investigación fue evaluado y aprobado por la Comisión Asesora en Investigación Biomédica en Seres Humanos (CAIBSH).

\section{RESULTADOS}

Se incluyeron 120 pacientes. La intervención fue exitosa en el $72 \%$ de los casos y se registró falla en el $28 \%$ de los casos.

En la Tabla 1, se muestran las variables demográficas y clínicas de los pacientes enrolados antes de la conexión a CPAP y se comparan aquellos que tuvieron éxito versus los que fracasaron.

Se realizaron estudios de IFI a todos los pacientes, que detectaron virus sincicial respiratorio (VSR) en 70 casos. En el 5\%, se detectó H1N1.

Se utilizaron presiones de CPAP de 5 a $8 \mathrm{~cm}$ de agua, con un promedio de $6 \mathrm{~cm}$ de agua.
Un $3,3 \%$ de los casos presentaron complicaciones (distención abdominal, neumotórax).

La duración media de la CPAP de burbuja fue de 74,93 h (IC 95\%: 65,22-84,65).

La media de días de internación en Cuidados Intermedios fue de 10 días (IC 95\%: 9,11-10,91); en el grupo de éxito, fue de 8,33 (DE 3,36) en Cuidados Intermedios y de 14,93 días (DE 5,19) en el grupo de fracaso (en este último, se incluyó la estadía en Terapia Intensiva).

En la Tabla 2, se expresan las variables medidas durante las horas posteriores a la colocación de la CPAP de burbuja.

El análisis posterior identificó que un descenso del 15\% de la FR y de 2 puntos en el puntaje de Tal a las 2 horas de iniciada la ventilación no invasiva (VNI) eran los factores mejor relacionados con el éxito, con un OR 6,41 (IC 95\%: 2,68-15,36) y 9,07 (IC 95\%: 3,72-22,19), respectivamente.

Al combinar dos variables cualitativas, que eran el descenso de un 15\% en la FR y de 2 puntos en el puntaje de Tal a las 2 horas, el OR aumentó a 13,31 (IC 95\%: 4,51-39,26).

No hubo hallazgos estadísticamente significativos a las 24 y $48 \mathrm{~h}$.

TABLA 2. Resultados. Frecuencias respiratoria y cardiaca, $\mathrm{FiO}_{2}$, saturación de pulso y puntaje de Tal

\begin{tabular}{|c|c|c|c|c|c|c|c|}
\hline \multirow{2}{*}{$\begin{array}{r}\text { Variable } \\
\mathrm{h}\end{array}$} & \multicolumn{2}{|c|}{ Muestra completa } & \multicolumn{2}{|c|}{ Grupo de éxito } & \multicolumn{2}{|c|}{ Grupo de fracaso } & \multirow[t]{2}{*}{$\mathrm{p}$} \\
\hline & $\mathbf{n}$ & $\mathrm{X} \pm \mathrm{DE}$ & $\mathbf{n}$ & $\mathrm{X} \pm \mathrm{DE}$ & $\mathbf{n}$ & $\mathrm{X} \pm \mathrm{DE}$ & \\
\hline FR 2 & 120 & $55,7913,67$ & 86 & $51,78 \pm 11,3$ & 34 & $65,94 \pm 14,03$ & $<0,0001$ \\
\hline FR 6 & 114 & $55,72 \pm 13,9$ & 86 & $53,27 \pm 12,3$ & 28 & $63,25 \pm 15,94$ & 0,0008 \\
\hline FR 24 & 93 & $50,62 \pm 9,61$ & 86 & $50,17 \pm 9,4$ & 7 & $56,14 \pm 11,14$ & 0,1145 \\
\hline FR 48 & 84 & $48,73 \pm 10,21$ & 79 & $48,06 \pm 9,74$ & 5 & $59,20 \pm 13,01$ & 0,0171 \\
\hline FC 2 & 120 & $142,22 \pm 19,68$ & 86 & $139,06 \pm 18,01$ & 34 & $150,21 \pm 21,66$ & 0,0047 \\
\hline FC 6 & 114 & $140,46 \pm 19,37$ & 86 & $136,79 \pm 17,25$ & 28 & $151,75 \pm 21,40$ & 0,0003 \\
\hline FC 24 & 93 & $133,39 \pm 19,21$ & 86 & $132,84 \pm 19,38$ & 7 & $140,14 \pm 16,61$ & 0,3359 \\
\hline FC 48 & 84 & $127,42 \pm 21,98$ & 79 & $126,81 \pm 22,02$ & 5 & $137,00 \pm 21,12$ & 0,3177 \\
\hline $\mathrm{FiO}_{2} 2$ & 120 & $98,78 \pm 1,54$ & 86 & $98,95 \pm 1,37$ & 34 & $98,35 \pm 1,86$ & 0,0936 \\
\hline $\mathrm{FiO}_{2} 6$ & 115 & $98,89 \pm 1,63$ & 86 & $99,06 \pm 1,38$ & 29 & $98,38 \pm 2,18$ & 0,1238 \\
\hline $\mathrm{FiO}_{2} 24$ & 90 & $98,88 \pm 1,38$ & 82 & $98,93 \pm 1,34$ & 8 & $98,38 \pm 1,77$ & 0,2831 \\
\hline $\mathrm{FiO}_{2} 48$ & 83 & $98,36 \pm 2,14$ & 77 & $98,62 \pm 1,81$ & 6 & $95,00 \pm 3,35$ & 0,0470 \\
\hline Sat 2 & 119 & $86,5 \pm 15,26$ & 86 & $85,15 \pm 15,28$ & 33 & $90,03 \pm 14,85$ & 0,1188 \\
\hline Sat 6 & 114 & $82,73 \pm 17,66$ & 86 & $80,56 \pm 18,02$ & 28 & $89,39 \pm 14,92$ & 0,0208 \\
\hline Sat 24 & 87 & $72,26 \pm 18,32$ & 81 & $71,59 \pm 18,38$ & 6 & $81,33 \pm 16,08$ & 0,2108 \\
\hline Sat 48 & 80 & $66,25 \pm 16,93$ & 75 & $65,36 \pm 16,47$ & 5 & $79,6 \pm 20,02$ & 0,0682 \\
\hline Tal 2 & 120 & $6,08 \pm 2,02$ & 86 & $5,43 \pm 1,76$ & 34 & $7,74 \pm 1,69$ & $<0,0001$ \\
\hline Tal 6 & 114 & $5,54 \pm 1,83$ & 86 & $4,94 \pm 1,34$ & 28 & $7,39 \pm 1,91$ & $<0,0001$ \\
\hline
\end{tabular}

DE: desvío estándar; FR: frecuencia respiratoria; FC: frecuencia cardíaca; $\mathrm{FiO}_{2}$ : fracción inspirada de oxígeno;

Sat: saturación de oxígeno; Tal: puntaje de Tal; h: horas de inicio de ventilación no invasiva. 


\section{DISCUSIÓN}

En nuestro estudio, se describió el uso de CPAP de burbuja en pacientes menores de 2 años con IRAB, utilizado como estrategia de tratamiento para evitar la asistencia respiratoria mecánica convencional.

La intervención fue exitosa en el $72 \%$ de los casos y se registró falla en el $28 \%$. El porcentaje de éxito fue similar al porcentaje publicado por otros autores $(83 \%$ y $75,5 \%) .8,9$

En este trabajo, se identificó que, a las 2 horas de iniciada la VNI con CPAP de burbuja, un descenso del 15\% de la FR con respecto a la previa $\mathrm{y}$ un descenso de 2 puntos en el puntaje de Tal fueron los factores que mejor predijeron el éxito.

Otros autores también han identificado la disminución de la FR como predictora de éxito de la VNI. ${ }^{6}$ Esto determina la importancia del monitoreo de los parámetros clínicos y la evaluación continua de estos pacientes.

A diferencia de otros trabajos, ${ }^{8-11}$ en este estudio, se empleó el puntaje de Tal.

La escala clínica de Tal modificada ha demostrado ser muy útil en la práctica, ya que permite normalizar el manejo clínico y la toma de decisiones. ${ }^{12}$

Con respecto a la $\mathrm{FC}$, si bien disminuyó a las $2 \mathrm{~h}$ en forma significativa, esta variable se comportó como una buena predictora de falla recién a partir de las $6 \mathrm{~h}$, a diferencia de lo reportado por Mayordomo-Colunga et al., ${ }^{8}$ quienes mencionaron que la disminución de la FC en la primera hora era una buena predictora de éxito.

En este trabajo, no se encontraron diferencias estadísticamente significativas entre ambos grupos con respecto al peso, edad, factores predisponentes o diagnóstico de bronquiolitis o neumonía. MayordomoColunga et al. mencionaron, en su trabajo, la presencia de apneas, menor peso y menor edad como predictores de falla, ${ }^{8}$ como así también Antonelli et al. ${ }^{9}$ identificaron menor edad, mayor puntaje de Woods, síndrome de dificultad respiratoria aguda (SDRA) o neumonía.

En nuestra experiencia, la VNI administrada a través de CPAP de burbuja fue una estrategia con un alto porcentaje de éxito en niños con IRAB de moderada a grave.

\section{CONCLUSIÓN}

El uso de CPAP de burbuja precoz representa una estrategia que permite optimizar el acceso del paciente a cuidados de calidad y el aprovechamiento de los recursos disponibles.

En este trabajo, la FR, la FC a las 2 horas y el puntaje de Tal a las 2 y 6 horas posteriores al inicio de la VNI permitieron predecir el éxito o el fracaso de la técnica.

\section{REFERENCIAS}

1. Ministerio de Salud de la Nación. Estadísticas Vitales. [Consulta: 9 de enero de 2017]. Disponible en: http:// www.deis.msal.gov.ar/index.php/estadisticasvitales/.

2. Comité Nacional de Neumología, Comité Nacional de Infectología, Comité Nacional de Medicina Interna. Recomendaciones para el manejo de las infecciones respiratorias agudas bajas (IRAB) en menores de 2 años. Arch Argent Pediatr 2015;113(4):373-4.

3. Fortenberry JD, Del Toro J, Jefferson LS, Evey L, et al. Management of pediatric acute hypoxemic respiratory insufficiency with bilevel positive pressure (BiPAP) nasal mask ventilation. Chest 1995;108(4):1059-64.

4. Teague WG. Noninvasive ventilation in the pediatric intensive care unit for children whit acute respiratory failure. Pediatr Pilmonol 2003;35(6):418-26.

5. Medina Villanueva A, Prieto Espuñes S, Los Arcos Solas M, Rey Galán M, et al. Aplicación de la ventilación no invasiva en una unidad de cuidados intensivos pediátricos. An Pediatr (Barc) 2005;62(1):13-9.

6. Essouri S, Chevret l, Durand P, Haas V, et al. Noninvasive positive pressure ventilation: five years of experience in a pediatric intensive care unit. Pediatr Crit Care Med 2006;7(6):329-34.

7. Bernet V, Hug MI, Frey B. Predictive factors for the success of noninvasive mask ventilation in infants and children with acute respiratory failure. Pediatr Crit Care Med 2005; 6(6):660-4.

8. Mayordomo-Colunga J, Medina A, Rey C, Los Arcos M, et al. Predictores de éxito y de fracaso en la ventilación no invasiva en la bornquiolitis aguda. An Pediatr (Barc) 2009;708(1):34-9.

9. Larrar S, Essouri S, Durand P, Chevret L, et al. Place de la ventilation non invasive nasale dans la prise en charge des broncho-alvéolites sévéres. Arch Pediatr 2006;13(11): 1397-403.

10. Javouhey E, Barats A, Richard N, Stamm D, et al. Noninvasive ventilation as primary ventilatory support for infants with severe bronchiolitis. Intensive Care Med 2008;34(9):1608-14.

11. Antonelli M, Conti G, Moro M, Esquinas A, et al. Predictors of failure of noninvasive positive pressure ventilation in patients with acute hypoxemic respiratory failure: a multicenter study. Intensive Care Med 2001;27(11):1718-28.

12. Tal A, Bavilski C, Yohai D, Bearman J, et al. Dexamethasone and salbutamol in the treatment of acute wheezing in infants. Pediatrics 1983;71(1):13-8. 